\title{
Null Controllability of Some Systems of Two Backward Stochastic Heat Equations with One Control Force
}

\author{
Hongheng $\mathrm{Li}^{*}$ and Qi Lü ${ }^{\dagger}$
}

\begin{abstract}
In this paper, we establish the null controllability for system coupled by two backward stochastic parabolic equations. The desired controllability result is obtained by means of proving a suitable observability estimate for the dual system of the controlled system.
\end{abstract}

2000 Mathematics Subject Classification. Primary 93B05; Secondary 93B07

Key Words. Backward stochastic heat equations, null controllability.

\section{Introduction}

Let $T>0, G \subset \mathbb{R}^{n}(n \in \mathbb{N})$ be a given bounded domain with a $C^{4}$ boundary $\Gamma$, with $G_{0}$ a nonempty open subset of $G$. Put

$$
Q \triangleq(0, T) \times G, \quad \Sigma \triangleq(0, T) \times \Gamma
$$

Throughout this paper, we will use $C$ to denote a generic positive constant depending only on $G$ and $G_{0}$, which may change from line to line.

Let $\left(\Omega, \mathcal{F},\left\{\mathcal{F}_{t}\right\}_{t \geq 0}, P\right)$ be a complete filtered probability space on which a one dimensional standard Brownian motion $\{B(t)\}_{t \geq 0}$ is defined, such that $\left\{\mathcal{F}_{t}\right\}_{t \geq 0}$ is the natural filtration generated by $\{B(t)\}_{t \geq 0}$. Let $H$ be a Banach space. Denote by $L_{\mathcal{F}}^{2}(0, T ; H)$ the Banach space consisting of all $H$-valued $\left\{\mathcal{F}_{t}\right\}_{t \geq 0}$-adapted processes $X(\cdot)$ such that $\mathbb{E}\left(|X(\cdot)|_{L^{2}(0, T ; H)}^{2}\right)<\infty$,

*College of Mathematics and Information Science, Leshan Normal University, Leshan 614004, China. This work was partially supported by Sciencific Research Fond of Sichuan Provincial Education Department of China under grant 10ZC110, and the project Z1062 of Leshan Normal University of China. E-mail: hongheng. li@163.com.

${ }^{\dagger}$ Basque Center for Applied Mathematics (BCAM), Bizkaia Technology Park, Building 500, 48160, Derio, Basque Country, Spain; and School of Mathematical Sciences, University of Electronic Science and Technology of China, Chengdu 610054, China. This work was partially supported by the Grant MTM2008-03541 of the MICINN, Spain, Project PI2010-04 of the Basque Government, the ERC Advanced Grant FP7-246775 NUMERIWAVES and the ESF Research Networking Programme OPTPDE. E-mail: qlu@bcamath.org. 
with the canonical norm; by $L_{\mathcal{F}}^{\infty}(0, T ; H)$ the Banach space consisting of all $H$-valued $\left\{\mathcal{F}_{t}\right\}_{t \geq 0^{-}}$ adapted bounded processes; by $L_{\mathcal{F}}^{2}(\Omega ; C([0, T] ; H))$ the Banach space consisting of all $H$ valued $\left\{\mathcal{F}_{t}\right\}_{t \geq 0}$-adapted processes $X(\cdot)$ such that $\mathbb{E}\left(|X(\cdot)|_{C(0, T ; H)}^{2}\right)<\infty$, with the canonical norm.

This paper is devoted to the study of the null controllability for the following coupled backward stochastic heat equations:

$$
\begin{cases}d y=-\Delta y d t+\left(a_{1} y+a_{2} z+a_{3} Y\right) d t+Y d B(t) & \text { in } Q \\ d z=-\Delta z d t+\left(b_{1} y+b_{2} z+b_{3} Z+\chi_{G_{0}} f\right) d t+Z d B(t) & \text { in } Q \\ y=z=0 & \text { on } \Sigma \\ y(T)=y_{T}, z(T)=z_{T} & \text { in } G\end{cases}
$$

where

$$
\begin{aligned}
& a_{i} \in L_{\mathcal{F}}^{\infty}\left(0, T ; L^{\infty}(G)\right),(i=1,2), a_{3} \in L_{\mathcal{F}}^{\infty}\left(0, T ; W^{1, \infty}(G)\right), \\
& b_{i} \in L_{\mathcal{F}}^{\infty}\left(0, T ; L^{\infty}(G)\right),(i=1,2), b_{3} \in L_{\mathcal{F}}^{\infty}\left(0, T ; W^{1, \infty}(G)\right),
\end{aligned}
$$

and $\chi_{G_{0}}$ is the characteristic function of $G_{0}$. In system $(1.1),\left(y_{T}, z_{T}\right) \in L^{2}\left(\Omega, \mathcal{F}_{T}, P ; L^{2}(G) \times\right.$ $\left.L^{2}(G)\right)$ is the terminal state, $(y, z)$ is the state variable and $f \in L_{\mathcal{F}}^{2}\left(0, T ; L^{2}\left(G_{0}\right)\right)$ is the control variable. By duality analysis as in [12], we can establish the existence and uniqueness for the solutions of system (1.1) in the class of

$$
\begin{aligned}
(y, z, Y, Z) \in & \left(L_{\mathcal{F}}^{2}\left(\Omega ; C\left([0, T] ; L^{2}(G) \times L^{2}(G)\right)\right) \cap L_{\mathcal{F}}^{2}\left(0, T ; H_{0}^{1}(G) \times H_{0}^{1}(G)\right)\right) \\
& \times L_{\mathcal{F}}^{2}\left(0, T ; L^{2}(G) \times L^{2}(G)\right) .
\end{aligned}
$$

The null controllability of system (1.1) is formulated as follows:

Definition 1.1 System (1.1) is said to be null controllable at time $T>0$ if for any given $\left(y_{T}, z_{T}\right) \in L^{2}\left(\Omega, \mathcal{F}_{T}, P ; L^{2}(G) \times L^{2}(G)\right)$, one can find a control $f \in L_{\mathcal{F}}^{2}\left(0, T ; L^{2}\left(G_{0}\right)\right)$ such that the solution $(y, z)$ of system (1.1) satisfies $(y(0), z(0))=(0,0)$ in $G, P$-a.s.

There are a great many works on the controllability theory of deterministic heat equations and heat systems(see $[1,4,5,6,7,13]$ and the references therein). However, things are quite different in the stochastic case. To the best of our knowledge, $[2,9,10,11]$ are the only four published papers in which the null controllability for stochastic heat equations is studied. As far as we know, there is no published paper which is concerned with the null controllability of stochastic heat system.

Noting that we only act one control on system (1.1), it is reasonable to expect that the action of $z$ to $y$ will be sufficiently effective. Hence we put the following condition on $a_{2}$ :

Condition 1.1 There is a nonempty subdomain $G_{1} \subset G_{0}$ and a constant $\sigma>0$ such that $a_{2}(x, t) \geq \sigma$ or $a_{2}(x, t) \leq-\sigma$, a.e. $(x, t) \in G_{1} \times(0, T), P$-a.s.

In this paper, we prove the following result. 
Theorem 1.1 Let Condition 1.1 hold. For any terminal state $\left(y_{T}, z_{T}\right) \in L^{2}\left(\Omega, \mathcal{F}_{T}, P ; L^{2}(G) \times\right.$ $\left.L^{2}(G)\right)$, we can find a control $f \in L_{\mathcal{F}}^{2}\left(0, T ; L^{2}(G)\right)$ such that the solution of system (1.1) with this control satisfies that $(y(0), z(0))=(0,0)$ in $G, P$-a.s. Moreover, we have the following estimate for the control:

$$
|f|_{L_{\mathcal{F}}^{2}\left(0, T ; L^{2}(G)\right)} \leq C e^{C\left[T^{-4}\left(1+p^{2}\right)+T\left(1+p^{2}\right)\right]}\left|\left(y_{T}, z_{T}\right)\right|_{L^{2}\left(\Omega, \mathcal{F}_{0}, P ; L^{2}(G) \times L^{2}(G)\right)},
$$

with

$$
p \triangleq \sum_{i=1}^{2}\left(\left|a_{i}\right|_{L^{\infty}\left(0, T ; L^{\infty}(G)\right)}+\left|b_{i}\right|_{L^{\infty}\left(0, T ; L^{\infty}(G)\right)}\right)+\left|a_{3}\right|_{L^{\infty}\left(0, T, W^{1, \infty}(G)\right)}+\left|b_{3}\right|_{L^{\infty}\left(0, T, W^{1, \infty}(G)\right)} .
$$

By means of the classical dual argument( see [11] for example), the null controllability of system (1.1) can be reduced to the observability estimate for the following coupled forward stochastic heat equations:

$$
\begin{cases}d w=\Delta w d t-\left(a_{1} w+b_{1} v\right) d t-a_{3} w d B(t) & \text { in } Q, \\ d v=\Delta v d t-\left(a_{2} w+b_{2} v\right) d t-b_{3} v d B(t) & \text { in } Q, \\ w=v=0 & \text { on } \Sigma, \\ w(0)=w_{0}, v(0)=v_{0} & \text { in } G,\end{cases}
$$

where $\left(w_{0}, z_{0}\right) \in L^{2}\left(\Omega, \mathcal{F}_{0}, P ; L^{2}(G) \times L^{2}(G)\right)$. We refer to [3] for the well-posedness of system (1.4) under suitable assumptions in the class

$$
(w, v) \in L_{\mathcal{F}}^{2}\left(\Omega ; C\left([0, T] ; L^{2}(G) \times L^{2}(G)\right)\right) \cap L_{\mathcal{F}}^{2}\left(0, T ; H_{0}^{1}(G) \times H_{0}^{1}(G)\right) .
$$

In order to prove Theorem 1.1, we only need to derive the following observability estimate for system (1.4).

Theorem 1.2 Let Condition 1.1 hold. Then any solution of system (1.4) satisfies that

$$
|(w, v)|_{L^{2}\left(\Omega, \mathcal{F}_{T}, P ; L^{2}(G) \times L^{2}(G)\right)} \leq C e^{C\left[T^{-4}\left(1+p^{2}\right)+T\left(1+p^{2}\right)\right]}|v|_{L_{\mathcal{F}}^{2}\left(0, T ; L^{2}\left(G_{0}\right)\right)} .
$$

The idea for the proof of Theorem 1.2 comes from the proof of an analogous result of Theorem 1.2 for deterministic heat systems(see [7] for example). We construct a functional $A(t)$ (see Section 3 for the details) to connect the suitable norm of $w$ and $v$. The difference here is that we need to utilize Itô calculus for the computation. This will lead to some additional terms, compared with the deterministic case. Treating these additional terms is the main difficulty we need to overcome.

The rest of this paper is organized as follows. In Section 2, we give some preliminaries. In Section 3, we prove Theorem 1.2. At last, in Section 4, we prove Theorem 1.1. 


\section{Some Preliminaries}

This section is addressed to give some preliminaries. To begin with, we introduce the following function.

Let $G_{2}$ and $G_{3}$ be two nonempty open subsets of $G$ such that $\overline{G_{2}} \subset G_{1}$ and $\overline{G_{3}} \subset G_{2}$. From Lemma 5.1 in [11], we know that there is a $\psi \in C^{4}(\bar{G})$ such that

$$
\begin{cases}\psi>0 & \text { in } G, \\ \psi=0 & \text { on } \partial G, \\ |\nabla \psi|>0 & \text { for all } x \in G \backslash G_{3} .\end{cases}
$$

Put

$$
\alpha(t, x)=\frac{e^{\lambda \psi(x)}-e^{2 \lambda|\psi|_{L} \infty(G)}}{t^{2}(T-t)^{2}}, \quad \varphi(t, x)=\frac{e^{\lambda \psi(x)}}{t^{2}(T-t)^{2}} .
$$

We have the following lemma for the observability estimate of backward stochastic heat equations.

Lemma 2.1 [11, Theorem 5.1] For any $T>0$, there is a constant $\lambda_{0}=\lambda_{0}\left(G, G_{2}\right)>0$ such that for all $\lambda \geq \lambda_{0}$, one can find two constants $C=C(\lambda)>0$ and $s_{0}=s_{0}(\lambda)>0$ so that for all $p \in L_{\mathcal{F}}^{2}\left(\Omega ; C\left([0, T] ; L^{2}(G)\right)\right) \cap L_{\mathcal{F}}^{2}\left(0, T ; H_{0}^{1}(G)\right), f \in L_{\mathcal{F}}^{2}\left(0, T ; L^{2}(G)\right)$ and $g \in L_{\mathcal{F}}^{2}\left(0, T ; H^{1}(G)\right)$ satisfying

$$
d p-\Delta p d t=f d t+g d B(t)
$$

and all $s \geq s_{1}=s_{1}(\lambda, T) \triangleq s_{0}(\lambda) \max \left(1, T^{2}\right)$, it holds that

$$
\begin{aligned}
& s^{3} \lambda^{4} \mathbb{E} \int_{Q} \varphi^{3} e^{2 s \alpha} p^{2} d x d t+s \lambda^{2} \mathbb{E} \int_{Q} \varphi e^{2 s \alpha}|\nabla p|^{2} d x d t \\
\leq & C\left\{\mathbb{E} \int_{Q} e^{2 s \alpha} f^{2} d x d t+s^{3} \lambda^{4} \mathbb{E} \int_{0}^{T} \int_{G_{2}} \varphi^{3} e^{2 s \alpha} p^{2} d x d t+s \lambda^{2} \mathbb{E} \int_{Q} \varphi e^{2 s \alpha} g^{2} d x d t\right. \\
& \left.+\mathbb{E} \int_{Q} \varphi e^{2 s \alpha} \sum_{i=1}^{n}\left[\left(g_{x_{i}}+s^{2} \alpha_{x_{i}} g\right)^{2}-\left(s \alpha_{x_{i}}^{2}+s \alpha_{x_{i} x_{i}}\right) g^{2}\right] d x d t\right\}
\end{aligned}
$$

Applying Lemma 2.1 to the first and second equation in system (1.4) respectively, we obtain that

$$
\begin{aligned}
& s^{3} \lambda^{4} \mathbb{E} \int_{Q} \varphi^{3} e^{2 s \alpha} w^{2} d x d t+s \lambda^{2} \mathbb{E} \int_{Q} \varphi e^{2 s \alpha}|\nabla w|^{2} d x d t \\
\leq & C\left\{\mathbb{E} \int_{Q} e^{2 s \alpha}\left(a_{1} w+b_{1} v\right)^{2} d x d t+s^{3} \lambda^{4} \mathbb{E} \int_{0}^{T} \int_{G_{2}} \varphi^{3} e^{2 s \alpha} w^{2} d x d t+s \lambda^{2} \mathbb{E} \int_{Q} \varphi e^{2 s \alpha}\left(a_{3} w\right)^{2} d x d t\right. \\
& \left.+\mathbb{E} \int_{Q} \varphi e^{2 s \alpha} \sum_{i=1}^{n}\left[\left(\left(a_{3} w\right)_{x_{i}}+s^{2} \alpha_{x_{i}}\left(a_{3} w\right)\right)^{2}-\left(s \alpha_{x_{i}}^{2}+s \alpha_{x_{i} x_{i}}\right)\left(a_{3} w\right)^{2}\right] d x d t\right\}
\end{aligned}
$$


and that

$$
\begin{aligned}
& s^{3} \lambda^{4} \mathbb{E} \int_{Q} \varphi^{3} e^{2 s \alpha} v^{2} d x d t+s \lambda^{2} \mathbb{E} \int_{Q} \varphi e^{2 s \alpha}|\nabla v|^{2} d x d t \\
\leq & C\left\{\mathbb{E} \int_{Q} e^{2 s \alpha}\left(a_{1} w+b_{1} v\right)^{2} d x d t+s^{3} \lambda^{4} \mathbb{E} \int_{0}^{T} \int_{G_{2}} \varphi^{3} e^{2 s \alpha} v^{2} d x d t+s \lambda^{2} \mathbb{E} \int_{Q} \varphi e^{2 s \alpha}\left(a_{3} w\right)^{2} d x d t\right. \\
& \left.+\mathbb{E} \int_{Q} \varphi e^{2 s \alpha} \sum_{i=1}^{n}\left[\left(\left(b_{3} v\right)_{x_{i}}+s^{2} \alpha_{x_{i}}\left(b_{3} v\right)\right)^{2}-\left(s \alpha_{x_{i}}^{2}+s \alpha_{x_{i} x_{i}}\right)\left(b_{3} v\right)^{2}\right] d x d t\right\} .
\end{aligned}
$$

By means of inequality (2.5) and inequality (2.6), choosing

$$
s \geq s_{2} \triangleq \max \left\{p^{\frac{2}{3}}, s_{1}\right\}
$$

we get that

$$
\mathbb{E} \int_{Q} \varphi^{3} e^{2 s \alpha}\left(w^{2}+v^{2}\right) d x d t \leq C \mathbb{E} \int_{0}^{T} \int_{G_{2}} \varphi^{3} e^{2 s \alpha}\left(w^{2}+v^{2}\right) d x d t .
$$

Hence we obtain the following proposition.

Proposition 2.1 Let $(w, v)$ be a solution of system (1.4), then for each $\lambda \geq \lambda_{0}$ and all $s \geq s_{2}$, inequality (2.7) holds.

\section{Proof of Theorem 1.2}

In this section, we give a proof of Theorem 1.2.

Proof of Theorem 1.2: From Condition 1.1, we know that $a_{2}(x, t) \geq \sigma$ or $a_{2}(x, t) \leq-\sigma$, a.e. $(x, t) \in G_{1} \times(0, T), P$-a.s. Without loss of generality, we assume that $a_{2}(x, t) \leq-\sigma$, a.e. $(x, t) \in G_{1} \times(0, T), P$-a.s.

By the definition of $\alpha$, we know that

$$
s^{3} \lambda^{4} \mathbb{E} \int_{Q} \varphi^{3}\left(w^{2}+v^{2}\right) d x d t \leq C \mathbb{E} \int_{0}^{T} \int_{G_{2}} e^{\frac{5}{3} s \alpha}\left(w^{2}+v^{2}\right) d x d t
$$

Let $\xi \in C^{\infty}\left(\mathbb{R}^{n}\right)$ be a cut-off function satisfying that

$$
\xi=1 \text { in } G_{2}, \quad \xi=0 \text { in } \mathbb{R}^{n} \backslash G_{1}, \quad 0 \leq \xi \leq 1 \text { in } G_{1} .
$$

Put $\eta=\xi^{6}$. Let $\beta_{0}, \beta_{1}, k, l$ be positive numbers, which will be specified later.

Let

$$
A(t) \triangleq \mathbb{E} \int_{G}\left(e^{k \tau \alpha} \eta^{\frac{4}{3}} w^{2}+\beta_{0} e^{2 \tau \alpha} \eta w v+\beta_{1} e^{l \tau \alpha} \eta^{\frac{2}{3}} v^{2}\right) d x .
$$

Then we have that

$$
\begin{aligned}
d A(t)=\mathbb{E} \int_{G}\{ & k \tau e^{k \tau \alpha} \alpha_{t} \eta^{\frac{4}{3}} w^{2} d t+2 e^{k \tau \alpha} \eta^{\frac{4}{3}} w d w+e^{k \tau \alpha} \eta^{\frac{4}{3}}(d w)^{2} \\
& +2 \tau \beta_{0} e^{2 \tau \alpha} \alpha_{t} \eta w v d t+\beta_{0} e^{2 \tau \alpha} \eta(v d w+w d v+d w d v) \\
& \left.+\beta_{1} l \tau e^{l \tau \alpha} \alpha_{t} \eta^{\frac{2}{3}} v^{2} d t+2 \beta_{1} e^{l \tau} \eta^{\frac{2}{3}} v d v+\beta_{1} e^{l \tau \alpha} \eta^{\frac{2}{3}}(d v)^{2}\right\} d x
\end{aligned}
$$


Noting that $(w, v)$ is the solution of system (1.4), from equality (3.4), we obtain that

$$
\begin{aligned}
d A(t)=\mathbb{E} \int_{G} & \left\{k \tau e^{k \tau \alpha} \alpha_{t} \eta^{\frac{4}{3}} w^{2} d t+2 e^{k \tau \alpha} \eta^{\frac{4}{3}} w\left(\Delta w-a_{1} w-b_{1} v\right) d t+e^{k \tau \alpha} \eta^{\frac{4}{3}}\left(a_{3} w\right)^{2} d t\right. \\
& +\beta_{0} e^{2 \tau \alpha} \eta\left[2 \tau \alpha_{t} w v+v\left(\Delta w-a_{1} w-b_{1} v\right)+w\left(\Delta v-a_{2} w-b_{2} v\right)+a_{3} w b_{3} v\right] d t \\
& \left.+\beta_{1} l \tau e^{l \tau \alpha} \alpha_{t} \eta^{\frac{2}{3}} v^{2} d t+\beta_{1} e^{l \tau \alpha} \eta^{\frac{2}{3}} v\left(\Delta v-a_{2} w-b_{2} v\right) d t+\beta_{1} e^{l \tau \alpha} \eta^{\frac{2}{3}}\left(b_{3} v\right)^{2} d t\right\} .
\end{aligned}
$$

Integrating equality $(3.5)$ in $[0, T]$, we get that

$$
\begin{aligned}
0= & -\beta_{0} \mathbb{E} \int_{Q} e^{2 \tau \alpha} \eta a_{2} w^{2} d x d t+\mathbb{E} \int_{Q}\left(k \tau e^{k \tau \alpha} \alpha_{t} \eta^{\frac{4}{3}} w^{2}-2 e^{k \tau \alpha} \eta^{\frac{4}{3}} a_{1} w^{2}+e^{k \tau \alpha} \eta^{\frac{4}{3}} a_{3}^{2} w^{2}\right) d x d t \\
& -\mathbb{E} \int_{Q}\left(2 e^{k \tau \alpha} \eta^{\frac{4}{3}} b_{1} w v-2 \beta_{0} \tau e^{2 \tau \alpha} \alpha_{t} \eta w v+\beta_{0} e^{2 \tau \alpha} \eta\left(a_{1}+b_{2}-a_{3} b_{3}\right) w v+2 \beta_{1} e^{l \tau \alpha} \eta^{\frac{2}{3}} a_{2} w v\right) d x d t \\
& +\mathbb{E} \int_{Q}\left(\beta_{1} l \tau e^{l \tau \alpha} \alpha_{t} \eta^{\frac{2}{3}} v^{2}-\beta_{0} e^{2 \tau \alpha} \eta b_{1} v^{2}-2 \beta_{1} e^{l \tau \alpha} \eta^{\frac{2}{3}} b_{2} v^{2}+\beta_{1} e^{l \tau \alpha} \eta^{\frac{2}{3}} b_{3}^{2} v^{2}\right) d x d t \\
& +\mathbb{E} \int_{Q}\left(2 e^{k \tau \alpha} \eta^{\frac{4}{3}} w \Delta w+\beta_{0} e^{2 \tau \alpha} \eta(v \Delta w+w \Delta v)+2 \beta_{1} e^{l \tau \alpha} \eta^{\frac{2}{3}} v \Delta v\right) d x d t .
\end{aligned}
$$

Denoting by $I_{i}(i=1,2,3,4)$ the last four terms on the right-hand side of equality $(3.6)$, we obtain that

$$
\beta_{0} \mathbb{E} \int_{Q} a_{2} e^{2 \tau \alpha} \eta w^{2} d x d t=I_{1}+I_{2}+I_{3}+I_{4} .
$$

Now we are going to estimate $I_{i}(i=1,2,3,4)$.

Choosing $k>2, r \in\left[\frac{3}{2}, 2\right), l>1+\frac{r}{2}$, by the definition of $\alpha$, we know that there is a $s_{3}>0$ such that for all $s \geq s_{3}$, it holds that

$$
\begin{array}{ll}
\left|k \tau e^{(k-2) \tau \alpha} \alpha_{t}\right|_{L^{\infty}(Q)} \leq 1, & \left|e^{(k-2) \tau \alpha}\right|_{L^{\infty}(Q)} \leq 1, \quad\left|l \tau e^{(l-r) \tau \alpha} \alpha_{t}\right|_{L^{\infty}(Q)} \leq 1, \\
\left|e^{(2-r) \tau \alpha}\right|_{L^{\infty}(Q)} \leq 1, \quad & \left|e^{(l-r) \tau \alpha}\right|_{L^{\infty}(Q)} \leq 1, \quad\left|e^{\left(k-1-\frac{r}{2}\right) \tau \alpha}\right|_{L^{\infty}(Q)} \leq 1, \\
\left|e^{\left(1-\frac{r}{2}\right) \tau \alpha} \alpha_{t}\right|_{L^{\infty}(Q)} \leq 1, & \left|e^{\left(1-\frac{r}{2}\right) \tau \alpha}\right|_{L^{\infty}(Q)} \leq 1, \quad\left|e^{\left(l-1-\frac{r}{2}\right) \tau \alpha}\right|_{L^{\infty}(Q)} \leq 1, \\
|\tau| \nabla \alpha\left|e^{\frac{k-2}{2} \tau \alpha}\right|_{L^{\infty}(Q)} \leq 1, & \left|\tau \varphi e^{(k-2) \tau \alpha}\right|_{L^{\infty}(Q)} \leq 1 .
\end{array}
$$

By virtue of the first and second inequality in (3.8), we know that

$$
\begin{aligned}
I_{1} & =\mathbb{E} \int_{Q}\left(k \tau e^{k \tau \alpha} \alpha_{t} \eta^{\frac{4}{3}} w^{2}-2 e^{k \tau \alpha} \eta^{\frac{4}{3}} a_{1} w^{2}+e^{k \tau \alpha} \eta^{\frac{4}{3}} a_{3}^{2} w^{2}\right) d x d t \\
& =\mathbb{E} \int_{Q} e^{2 \tau \alpha}\left(k \tau e^{(k-2) \tau \alpha} \alpha_{t} \eta^{\frac{4}{3}} w^{2}-2 e^{(k-2) \tau \alpha} \eta^{\frac{4}{3}} a_{1} w^{2}+e^{(k-2) \tau \alpha} \eta^{\frac{4}{3}} a_{3}^{2} w^{2}\right) d x d t \\
& \leq C\left(p+p^{2}+1\right) \mathbb{E} \int_{Q} e^{2 \tau \alpha} \eta w^{2} d x d t .
\end{aligned}
$$

As the estimate of $I_{1}$, by the third, the fourth and the fifth inequality in (3.8), one can easily obtain that

$$
I_{3}=\mathbb{E} \int_{Q}\left(\beta_{1} l \tau e^{l \tau \alpha} \alpha_{t} \eta^{\frac{2}{3}} v^{2}-\beta_{0} e^{2 \tau \alpha} \eta b_{1} v^{2}-2 \beta_{1} e^{l \tau \alpha} \eta^{\frac{2}{3}} b_{2} v^{2}+\beta_{1} e^{l \tau \alpha} \eta^{\frac{2}{3}} b_{3}^{2} v^{2}\right) d x d t
$$




$$
\begin{aligned}
& =\mathbb{E} \int_{Q} e^{r \tau \alpha} \eta^{\frac{1}{3}} v^{2}\left(\beta_{1} l \tau e^{(l-r) \tau \alpha} \alpha_{t} \eta^{\frac{2}{3}}-\beta_{0} e^{(2-r) \tau \alpha} \eta b_{1}-2 \beta_{1} e^{(l-r) \tau \alpha} \eta^{\frac{2}{3}} b_{2}+\beta_{1} e^{(l-r) \tau \alpha} \eta^{\frac{2}{3}} b_{3}^{2}\right) d x d t \\
& \leq C\left[\left(\beta_{0}+\beta_{1}\right)\left(p+p^{2}\right)+\beta_{1}\right] \mathbb{E} \int_{Q} e^{r \tau \alpha} \eta^{\frac{1}{3}} v^{2} d x d t
\end{aligned}
$$

Now we estimate $I_{2}$. By Cauchy-Schwartz inequality, utilizing the sixth, the seventh, the eighth and the ninth inequality in (3.8), we have

$$
\begin{aligned}
I_{2}= & -\mathbb{E} \int_{Q}\left[2 e^{k \tau \alpha} \eta^{\frac{4}{3}} b_{1} w v-2 \beta_{0} \tau e^{2 \tau \alpha} \alpha_{t} \eta w v+\beta_{0} e^{2 \tau \alpha} \eta\left(a_{1}+b_{2}-a_{3} b_{3}\right) w v\right. \\
& \left.\quad+2 \beta_{1} e^{l \tau \alpha} \eta^{\frac{2}{3}} a_{2} w v\right] d x d t \\
\leq & \frac{1}{4} \mathbb{E} \int_{Q} e^{2 \tau \alpha} \eta w^{2} d x d t+\mathbb{E} \int_{Q} e^{r \tau \alpha} \eta^{\frac{1}{3}} v^{2}\left[2 e^{\left(k-1-\frac{r}{2}\right) \tau \alpha} \eta^{\frac{2}{3}} b_{1}-2 \tau \beta_{0} e^{\left(1-\frac{r}{2}\right) \tau \alpha} \alpha_{t} \eta^{\frac{1}{3}}\right. \\
& \left.+\beta_{0} e^{\left(1-\frac{r}{2}\right) \tau \alpha} \eta^{\frac{1}{3}}\left(a_{1}+b_{2}-a_{3} b_{3}\right)+2 \beta_{1} e^{\left(l-1-\frac{r}{2}\right) \tau \alpha} a_{2}\right]^{2} d x d t .
\end{aligned}
$$

Recalling that $l>1+\frac{r}{2}$ and noticing that $1+\frac{r}{2}>r$, we obtain that

$$
I_{2} \leq C\left[\left(\beta_{0}+\beta_{1}\right)\left(p+p^{2}\right)\right] \mathbb{E} \int_{Q} e^{r \tau \alpha} \eta^{\frac{1}{3}} v^{2} d x d t+\frac{1}{4} \mathbb{E} \int_{Q} e^{2 \tau \alpha} \eta w^{2} d x d t .
$$

At last, we estimate $I_{4}$.

$$
\begin{aligned}
I_{4}= & \mathbb{E} \int_{Q}\left(2 e^{k \tau \alpha} \eta^{\frac{4}{3}} w \Delta w+\beta_{0} e^{2 \tau \alpha} \eta(v \Delta w+w \Delta v)+2 \beta_{1} e^{l \tau \alpha} \eta^{\frac{2}{3}} v \Delta v\right) d x d t \\
= & \mathbb{E} \int_{Q} e^{k \tau \alpha} \eta^{\frac{4}{3}} \Delta w^{2} d x d t-2 \mathbb{E} \int_{Q} e^{k \tau \alpha} \eta^{\frac{4}{3}}|\nabla w|^{2} d x d t \\
& +\beta_{0} \mathbb{E} \int_{Q} e^{2 \tau \alpha} \eta \Delta(w v) d x d t-2 \beta_{0} \mathbb{E} \int_{Q} e^{2 \tau \alpha} \eta \nabla w \cdot \nabla v d x d t \\
& +\beta_{1} \mathbb{E} \int_{Q} e^{l \tau \alpha} \eta^{\frac{2}{3}} \Delta v^{2} d x d t-2 \beta_{1} \mathbb{E} \int_{Q} e^{l \tau \alpha} \eta^{\frac{2}{3}}|\nabla v|^{2} d x d t .
\end{aligned}
$$

By virtue of integration by parts, we get that

$$
\begin{aligned}
& \mathbb{E} \int_{Q} e^{k \tau \alpha} \eta^{\frac{4}{3}} \Delta w^{2} d x d t \\
= & \mathbb{E} \int_{Q} \Delta\left(e^{k \tau \alpha} \eta^{\frac{4}{3}}\right) w^{2} d x d t \\
= & \mathbb{E} \int_{Q} e^{k \tau \alpha} w^{2}\left(k^{2} \tau^{2}|\nabla \alpha|^{2} \eta^{\frac{4}{3}}+k \tau \Delta \alpha \eta^{\frac{4}{3}}+\frac{4}{3}|\nabla \eta|^{2} \eta^{-\frac{2}{3}}+\frac{4}{3} \eta^{\frac{1}{3}} \Delta \eta+\frac{8}{3} k \tau \eta^{\frac{1}{3}} \nabla \alpha \cdot \nabla \eta\right) .
\end{aligned}
$$

It is easy to check that

$$
\eta^{-\frac{5}{6}} \nabla \eta=6 \nabla \xi \in L^{\infty}(Q), \quad \eta^{-\frac{2}{3}} \Delta \eta=30|\nabla \xi|^{2}+6 \xi \Delta \xi \in L^{\infty}(Q) .
$$


Recalling $k>2$, by means of the last two inequalities in (3.8), we can obtain

$$
\mathbb{E} \int_{Q} e^{k \tau \alpha} \eta^{\frac{4}{3}} \Delta w^{2} d x d t \leq C \mathbb{E} \int_{Q} e^{2 \tau \alpha} \eta w^{2} d x d t .
$$

With the similar argument to obtain inequality (3.12) and inequality (3.16), we can show that

$$
\beta_{0} \mathbb{E} \int_{Q} e^{2 \tau \alpha} \eta \Delta(w v) d x d t \leq \frac{1}{4} \mathbb{E} \int_{Q} e^{2 \tau \alpha} \eta w^{2} d x d t+C \beta_{0}^{2} \mathbb{E} \int_{Q} e^{r \tau \alpha} \eta^{\frac{1}{3}} v^{2} d x d t
$$

and that

$$
\beta_{1} \mathbb{E} \int_{Q} e^{l \tau \alpha} \eta^{\frac{2}{3}} \Delta v^{2} d x d t \leq C \beta_{1} \mathbb{E} \int_{Q} e^{r \tau \alpha} \eta^{\frac{1}{3}} v^{2} d x d t
$$

Then it follows from (3.13)-(3.18) that,

$$
\begin{aligned}
I_{4} \leq & C \mathbb{E} \int_{Q} e^{2 \tau \alpha} \eta w^{2} d x d t+C\left(\beta_{0}^{2}+\beta_{1}\right) \mathbb{E} \int_{Q} e^{r \tau \alpha} \eta^{\frac{1}{3}} v^{2} d x d t \\
& -2 \mathbb{E} \int_{Q} e^{k \tau \alpha} \eta^{\frac{4}{3}}|\nabla w|^{2} d x d t-2 \beta_{0} \mathbb{E} \int_{Q} e^{2 \tau \alpha} \eta \nabla w \cdot \nabla v d x d t \\
& -2 \beta_{1} \mathbb{E} \int_{Q} e^{l \tau \alpha} \eta^{\frac{2}{3}}|\nabla v|^{2} d x d t .
\end{aligned}
$$

Let $k+l<4$ and $\beta_{1}>\frac{\beta_{0}^{2}}{4}$, then we know

$$
-2 \mathbb{E} \int_{Q} e^{k \tau \alpha} \eta^{\frac{4}{3}}|\nabla w|^{2} d x d t-2 \beta_{0} \mathbb{E} \int_{Q} e^{2 \tau \alpha} \eta \nabla w \cdot \nabla v d x d t-2 \beta_{1} \mathbb{E} \int_{Q} e^{l \tau \alpha} \eta^{\frac{2}{3}}|\nabla v|^{2} d x d t \leq 0 .
$$

Therefore we find

$$
I_{4} \leq C \mathbb{E} \int_{Q} e^{2 \tau \alpha} \eta w^{2} d x d t+C\left(\beta_{0}^{2}+\beta_{1}\right) \mathbb{E} \int_{Q} e^{r \tau \alpha} \eta^{\frac{1}{3}} v^{2} d x d t .
$$

From (3.9)-(3.21), we see

$$
\begin{aligned}
\beta_{0} \mathbb{E} \int_{Q} a_{2} e^{2 \tau \alpha} \eta w^{2} d x d t \leq & C\left(1+p^{2}\right)\left(\beta_{0}^{2}+\beta_{1}^{2}\right) \mathbb{E} \int_{Q} e^{r \tau \alpha} \eta^{\frac{1}{3}} v^{2} d x d t \\
& +C\left(p^{2}+1\right) \int_{Q} e^{2 \tau \alpha} \eta w^{2} d x d t
\end{aligned}
$$

Finally, by setting $\beta_{0}=2 C\left(1+p^{2}\right)$, we obtain that

$$
\mathbb{E} \int_{Q} e^{2 \tau \alpha} \eta w^{2} d x d t \leq C\left(1+p^{10}\right) \mathbb{E} \int_{Q} e^{r \tau \alpha} \eta^{\frac{1}{3}} v^{2} d x d t
$$


Taking into account of Proposition 2.1, inequality (3.1) and inequality (3.23), for $\lambda \geq \lambda_{0}$ and $s \geq \max \left\{s_{2}, s_{3}\right\}$, we deduce that

$$
\mathbb{E} \int_{Q} \varphi^{3} e^{2 s \alpha}\left(w^{2}+v^{2}\right) d x d t \leq C\left(1+p^{10}\right) \mathbb{E} \int_{0}^{T} \int_{G_{0}} e^{\frac{3}{2} s \alpha} v^{2} d x d t .
$$

Recalling the definition of $\alpha$ and $\phi$ (see (2.2)), we have that

$$
\mathbb{E} \int_{Q} \varphi^{3} e^{2 s \alpha}\left(w^{2}+v^{2}\right) d x d t \geq \min _{x \in G}\left[\varphi^{3}\left(x, \frac{T}{2}\right) e^{2 s \alpha\left(x, \frac{T}{2}\right)}\right] \mathbb{E} \int_{\frac{T}{4}}^{\frac{3 T}{4}} \int_{G}\left(w^{2}+v^{2}\right) d x d t,
$$

and that

$$
\mathbb{E} \int_{0}^{T} \int_{G_{0}} e^{\frac{3}{2} s \alpha} v^{2} d x d t \leq \max _{(x, t) \in \bar{Q}}\left(e^{\frac{3}{2} s \alpha(x, t)}\right) \mathbb{E} \int_{0}^{T} \int_{G_{0}} v^{2} d x d t
$$

From (3.24)-(3.26), we obtain that

$$
\mathbb{E} \int_{\frac{T}{4}}^{\frac{3 T}{4}} \int_{G}\left(w^{2}+v^{2}\right) d x d t \leq C e^{C T^{-4}\left(1+p^{2}\right)} \mathbb{E} \int_{0}^{T} \int_{G_{0}} v^{2} d x d t .
$$

Noting that $d\left(w^{2}+v^{2}\right)=2 w d w+(d w)^{2}+2 v d v+(d v)^{2}$, applying the usual energy estimate to system (1.4), it is easy to see that, for any $0 \leq t_{1} \leq t_{2} \leq T$, it holds

$$
\begin{aligned}
& \mathbb{E} \int_{G}\left[w^{2}\left(t_{2}\right)+v^{2}\left(t_{2}\right)\right] d x-\mathbb{E} \int_{G}\left[w^{2}\left(t_{1}\right)+v^{2}\left(t_{1}\right)\right] d x \\
= & \mathbb{E} \int_{t_{1}}^{t_{2}} \int_{G}\left[2 w d w+(d w)^{2}+2 v d v+(d v)^{2}\right] d x d t \\
= & \mathbb{E} \int_{t_{1}}^{t_{2}} \int_{G}\left[2 w\left(\Delta w-a_{1} w-b_{1} v\right)+\left(a_{3} w\right)^{2}+2 v\left(\Delta v-a_{2} w-b_{2} v\right)+\left(b_{3} v\right)^{2}\right] d x d t \\
\leq & C\left(1+p^{2}\right) \mathbb{E} \int_{t_{1}}^{t_{2}} \int_{G}\left(w^{2}+v^{2}\right) d x d t
\end{aligned}
$$

Hence, in terms of Gronwall inequality, it follows

$$
\mathbb{E} \int_{G}\left[w^{2}\left(t_{2}\right)+v^{2}\left(t_{2}\right)\right] d x \leq e^{C T\left(1+p^{2}\right)} \mathbb{E} \int_{G}\left[w^{2}\left(t_{1}\right)+v^{2}\left(t_{1}\right)\right] d x .
$$

By inequality (3.27) and inequality (3.29), we conclude that the solution $(w, v)$ of system (1.4) satisfies inequality (1.5).

\section{Proof of Theorem 1.1}

This section is devoted to the proof of our controllability results: Theorems 1.1. The proof is almost standard dual argument. However, for the sake of completeness, we still give it here. 
Proof of Theorem 1.1: For any $\left(y_{T}, z_{T}\right) \in L^{2}\left(\Omega, \mathcal{F}_{T}, P ; L^{2}(G) \times L^{2}(G)\right)$, we need to find a control $f \in L_{\mathcal{F}}^{2}\left(0, T ; L^{2}\left(G_{0}\right)\right)$ such that the solution of system (1.1) satisfies $(y(0), z(0))=$ $(0,0)$ in $G, P$-a.s. We use the duality argument.

We introduce the following linear subspace of $L_{\mathcal{F}}^{2}\left(0, T ; L^{2}\left(G_{0}\right)\right) \times L_{\mathcal{F}}^{2}\left(0, T ; L^{2}(G)\right)$ :

$$
\begin{aligned}
X \triangleq\left\{\left.v\right|_{[0, T] \times G_{0} \times \Omega} \mid\right. & (w, v) \text { solves system }(1.4) \text { with some } \\
& \left.\left(w_{0}, v_{0}\right) \in L^{2}\left(\Omega, \mathcal{F}_{0}, P ; L^{2}(G) \times L^{2}(G)\right)\right\},
\end{aligned}
$$

and define a linear functional on $X$ as follows:

$$
L\left(\left.v\right|_{[0, T] \times G_{0} \times \Omega}\right)=\mathbb{E} \int_{G}\left(y_{T} w(T)+z_{T} v(T)\right) d x .
$$

By means of the observability estimate ( see Theorem 1.2), we know that

$$
\begin{aligned}
\left|L\left(\left.v\right|_{[0, T] \times G_{0} \times \Omega}\right)\right| & \leq\left(\mathbb{E} \int_{G}\left(\left|y_{T}\right|^{2}+\left|z_{T}\right|^{2}\right) d x\right)^{\frac{1}{2}}\left(\mathbb{E} \int_{G}\left(|w(T)|^{2}+|v(T)|^{2}\right) d x\right)^{\frac{1}{2}} \\
& \leq C e^{C\left[T^{-4}\left(1+p^{2}\right)+T\left(1+p^{2}\right)\right]}\left(\mathbb{E} \int_{G}\left(\left|y_{T}\right|^{2}+\left|z_{T}\right|^{2}\right) d x\right)^{\frac{1}{2}}\left(\mathbb{E} \int_{0}^{T} \int_{G_{0}}|v|^{2} d x d t\right)^{\frac{1}{2}} .
\end{aligned}
$$

Thus, $L$ is a bounded linear functional on $X$ such that the norm of $l$ is bounded by

$$
C e^{C\left[T^{-4}\left(1+p^{2}\right)+T\left(1+p^{2}\right)\right]}\left(\mathbb{E} \int_{G}\left(\left|y_{T}\right|^{2}+\left|z_{T}\right|^{2}\right) d x\right)^{\frac{1}{2}} .
$$

By Hahn-Banach Theorem, $L$ can be extended to a bounded linear functional with the same norm on $L_{\mathcal{F}}^{2}\left(0, T ; L^{2}\left(G_{0}\right)\right)$. For simplicity, we use the same notation for this extension. Now, Riesz Representation Theorem allows us to find a random field $f \in L_{\mathcal{F}}^{2}\left(0, T ; L^{2}\left(G_{0}\right)\right)$ such that

$$
\mathbb{E} \int_{G}\left[y_{T} w(T)+z_{T} v(T)\right] d x=\mathbb{E} \int_{0}^{T} \int_{G_{0}} f v d x d t,
$$

and that

$$
|f|_{L_{\mathcal{F}}^{2}\left(0, T ; L^{2}(G)\right)} \leq C e^{C\left[T^{-4}\left(1+p^{2}\right)+T\left(1+p^{2}\right)\right]}\left|\left(y_{T}, z_{T}\right)\right|_{L^{2}\left(\Omega, \mathcal{F}_{0}, P ; L^{2}(G) \times L^{2}(G)\right)} .
$$

We claim that this random field $f$ is exactly the control we need. In fact, by means of Itô formula, we know that

$$
d(y w)=y d w+w d y+d y d w
$$

and that

$$
d(z v)=z d v+v d z+d z d v
$$

where $(y, z)$ is the solution to system $(1.1)$ and $(w, v)$ is the solution to system (1.4). From 
(4.3), we obtain that

$$
\begin{aligned}
& \mathbb{E} \int_{G} y_{T} w(T) d x-\mathbb{E} \int_{G} y(0) w_{0} d x \\
= & \mathbb{E} \int_{Q}(y d w+w d y+d y d w) d x \\
= & \mathbb{E} \int_{Q} y\left(\Delta w-a_{1} w-b_{1} v\right) d x d t+\mathbb{E} \int_{Q} w\left(-\Delta y+a_{1} y+a_{2} z+a_{3} Y\right) d x d t \\
& +\mathbb{E} \int_{Q} Y\left(-a_{3} w\right) d x d t \\
= & \mathbb{E} \int_{Q} a_{2} w z-b_{1} v y d x d t .
\end{aligned}
$$

From (4.3), we know that

$$
\begin{aligned}
& \mathbb{E} \int_{G} z_{T} v(T) d x-\mathbb{E} \int_{G} z(0) v_{0} d x \\
= & \mathbb{E} \int_{Q}(z d v+v d z+d z d v) d x \\
= & \mathbb{E} \int_{Q} z\left(\Delta v-a_{2} w-b_{2} v\right) d x d t+\mathbb{E} \int_{Q} v\left(-\Delta z+b_{1} y+b_{2} z+b_{3} Z+\chi_{G_{0}} f\right) d x d t \\
& +\mathbb{E} \int_{Q} Z\left(-b_{3} v\right) d x d t \\
= & \mathbb{E} \int_{Q} b_{1} v y-a_{2} w z+\chi_{G_{0}} f v d x d t,
\end{aligned}
$$

Combining equality (4.1), equality (4.5) and equality (4.6), we find

$$
\mathbb{E} \int_{G} y(0) w_{0} d x+\mathbb{E} \int_{G} z(0) v_{0} d x=0 .
$$

Since $\left(w_{0}, v_{0}\right)$ can be chosen arbitrarily, this implies that $(y(0), z(0))=0$ in $G, P$-a.s.

\section{References}

[1] V. Barbu, Controllability of parabolic and Navier-Stokes equations, Sci. Math. Jpn., 56(2002), 143-211.

[2] V. Barbu, A. Răscanu and G. Tessitore, Carleman estimate and cotrollability of linear stochastic heat equatons, Appl. Math. Optim., 47(2003), 97-120.

[3] G. Da Prato and J. Zabczyk, Stochastic Equations in Infinite Dimensions, Cambridge University Press, Cambridge, 1992.

[4] A. Doubova, E. Fernández-Cara, M. González-Burgos and E. Zuazua, On the controllability of parabolic systems with a nonlinear term involving the state and the gradient, SIAM J. Control Optim., 41(2002), 798-819.

[5] E. Fernandez-Cara and S. Guerrero, Global Carleman inequalities for parabolic systems and applications to controllability, SIAM J. Control Optim., 45 (2006), 1399-1446. 
[6] A. V. Fursikov and O. Yu. Imanuvilov, Controllability of Evolution Equations, Lecture Notes Series, 34, Seoul National University, Reserach Institute of Mathematics, Global Analysis Research Center, Seoul, 1996.

[7] S. Guerrero, Null controllability of some systems of two parabolic equations with one control force, SIAM J. Control Optim., 46(2007), 379-394.

[8] J. L. Lions, Exact controllability, stabilization and perturbations for distributed systems, SIAM Riview, 30(1988), 1-68.

[9] Q. Lü, Some results on the controllability of forward stochastic heat equations with control on the drift, J. Funct. Anal., 260(2011), 832-851.

[10] M. Sîrbu and G. Tessitore, Null controllability of an infinite dimensional SDE with state- and controldependent noise, Systems Control Lett., 44(2001), 385-394.

[11] S. Tang and X. Zhang, Null controllability for forward and backward stochastic parabolic equations, SIAM J. Control Optim., 48(2009), 2191-2216.

[12] X. Zhou, A duality analysis on stochastic partial differential equations, J. Funct. Anal., 103(1992), 275-293.

[13] E. Zuazua, Controllability and observability of partial differential equations: some results and open problems, in Handbook of Differential Equations: Evolutionary Differential Equations, vol. 3, Elsevier Science, 2006, 527-621. 